\title{
HOMOLOGY OF INTEGRAL UPPER-TRIANGULAR MATRICES
}

\author{
W. G. DWYER
}

\begin{abstract}
We calculate the homology of the multip'icative group of integral upper-triangular $n \times n$ matrices at all primes $p \geqslant n-1$.
\end{abstract}

1. Group homology. For any $\operatorname{ring} A$, let $\mathrm{Gl}_{n}(A)$ be the general linear group of invertible $n \times n$ matrices over $A$, and $U_{n}(A)$ the subgroup of upper-triangular matrices with ones on the diagonal. The purpose of this note is to prove Theorem 1.1, which gives a calculation of the Eilenberg-Mac Lane group homology of $U_{n}(\mathbf{Z})$ at all primes $p \geqslant n-1$. (This group homology is the same as the homology of the compact nilmanifold $U_{n}(\mathbf{R}) / U_{n}(\mathbf{Z})$.)

For any permutation $\sigma$ in the symmetric group $S_{n}$, let $M_{\sigma} \in \mathrm{Gl}_{n}(A)$ denote the matrix with the property that $M_{\sigma} b_{i}=b_{\sigma(i)}$, where $b_{i}$ is the column vector with one in the $i$ th coordinate and zero in the others. Let $U_{n}^{-}(A)$ be the group of lower-triangular matrices in $\mathrm{Gl}_{n}(A)$ with ones on the diagonal, and $U_{n}^{\sigma}$ the intersection

$$
\left(M_{\sigma}^{-1} U_{n}^{-}(A) M_{\sigma}\right) \cap U_{n}(A) .
$$

It is easy to see that $U_{n}^{\sigma}(A)$ is the subgroup of $U_{n}(A)$ given by matrices which have zero in row $i$, column $j$ whenever $i<j$ and $\sigma(i)<\sigma(j)$. In particular, each quotient $U_{n}^{\sigma}(\mathbf{R}) / U_{n}^{\sigma}(\mathbf{Z})$ is a compact nilmanifold, and therefore has a fundamental homology class, well defined up to choice of orientation. The image of this fundamental class in $H_{*}\left(U_{n}(\mathbf{Z})\right)$ under the embedding $U_{n}^{\sigma}(\mathbf{R}) / U_{n}^{\sigma}(\mathbf{Z}) \rightarrow U_{n}(\mathbf{R}) / U_{n}(\mathbf{Z})$ will be denoted $C_{\sigma}$.

REMARK. The dimension of the cyclic $C_{\sigma}$ is the cardinality of the set

$$
L(\sigma)=\{(i, j): 1 \leqslant i<j \leqslant n, \sigma(i)>\sigma(j)\} .
$$

We will denote this cardinality by $l(\sigma)$. Note that the permutation $\sigma$ is uniquely determined by the set $L(\sigma)$.

Let $\mathbf{Z}_{(p)}$ stand for the localization of the ring $\mathbf{Z}$ at the prime $p$.

1.1 TheOREM. If $p \geqslant n-1$, then $H_{*}\left(U_{n}(\mathbf{Z}), \mathbf{Z}_{(p)}\right)$ is the free $\mathbf{Z}_{(p)}$-module on the classes $C_{\sigma}, \sigma \in S_{n}$.

The proof of Theorem 1.1 is in two steps. Let $U_{n}$ denote $U_{n}(\mathbf{Z})$.

1.2 Proposition. The cycles $C_{\sigma}, \sigma \in S_{n}$, generate a free $\mathbf{Z}$-summand of $\boldsymbol{H}_{*}\left(U_{n}, \mathbf{Z}\right)$ of rank $n !$.

Received by the editors April 17, 1984.

1980 Mathematics Subject Classification. Primary 55R35; Secondary 55S30, 17B56, $20 \mathrm{H} 25$. 
Proof. For each $\sigma \in S_{n}$ with $k=l(\sigma)$, it is enough to produce a map $f_{\sigma}$ : $H_{k}\left(U_{n}, \mathbf{Z}\right) \rightarrow \mathbf{Z}$ such that

$$
\begin{aligned}
& f_{\sigma}\left(C_{\sigma}\right)= \pm 1, \quad \text { and } \\
& f_{\sigma}\left(C_{\tau}\right)=\varnothing, \quad \tau \neq \sigma, l(\tau)=l(\sigma)
\end{aligned}
$$

Let $\rho$ be the order-reversing permutation given by $\rho(i)=n+1-i$, and let $\pi=$ $\rho \sigma \rho^{-1}$. Since $l(\pi)+l(\sigma)=\operatorname{dim}\left(U_{n}(\mathbf{R}) / U_{n}(\mathbf{Z})\right)$, algebraic intersection with the cycle $C_{\pi}$ defines a map $f_{\sigma}: H_{k}\left(U_{n}, \mathbf{Z}\right) \rightarrow \mathbf{Z}$. Since the geometric intersection of $C_{\pi}$ and $C_{\sigma}$ is a single point, it is clear that $f_{\sigma}\left(C_{\sigma}\right)= \pm 1$. If $\tau \neq \sigma$ but $l(\tau)=l(\sigma)$, then $C_{\pi}$ and $C_{\tau}$ intersect cleanly $[9$, p. 37] in a positive-dimensional compact nilmanifold $X$. By the clean intersection formula [9, p. 38], $f_{\sigma}\left(C_{\tau}\right)$ is equal to the Euler characteristic of $X$, which is clearly zero.

Let $u_{n}=u_{n}(\mathbf{Z})$ be the Lie algebra of strictly upper-triangular matrices in $\mathrm{gl}_{n}(\mathbf{Z})$.

1.3 Proposition. If $p \geqslant n-1$, the Lie algebra homology $H_{*}\left(u_{n}, \mathbf{Z}_{(p)}\right)$ is a free $\mathbf{Z}_{(p)}$-module of total rank $n$ !.

The proof of this will occupy $\$ \S 2-5$.

REMARK. P. Kunkel has shown that if $p<n-1$, the Lie algebra homology $H_{*}\left(u_{n}, \mathbf{Z}_{(p)}\right)$ contains $p$-torsion.

Proof OF 1.1. The universal enveloping algebra of $u_{n}$ is the associated graded of $\mathbf{Z}\left[U_{n}\right]$ with respect to the filtration given by powers of the augmentation ideal $[7, \S 7$; 1] so that there is a spectral sequence $[5,4.5(\mathrm{i}) ; 10$, p. 20]

$$
\left\{E_{*, *}^{2}=H_{*}\left(u_{n}, \mathbf{Z}_{(p)}\right)\right\} \Rightarrow H_{*}\left(U_{n}, \mathbf{Z}_{(p)}\right)
$$

By 1.2, 1.3 and a counting argument, this spectral sequence collapses and shows that $H_{*}\left(U_{n}, \mathbf{Z}_{(p)}\right)$ is free of rank $n$ !. Proposition 1.2 then identifies the $n$ ! generators.

REMARK. The homology with real coefficients of the group $U_{n}(Z)$ is known by [8] to be isomorphic to the real Lie algebra homology $H_{*}\left(u_{n}(\mathbf{R})\right)$. This real Lie algebra homology has been computed classically by Bott and Kostant $[6,4]$. In $\$ \S 2-5$ we give a new proof of an integral strengthening of the Bott-Kostant result; this new proof is closely related to recent work of Friedlander and Parshall on modular Lie algebra homology, and many of our results can be derived from theirs.

REMARK. This work was motivated by some exploratory computer calculations of Lie algebra homology carried out by Evens and Priddy. The Koszul complex grading described in $\$ 3$ can be used to speed up these calculations quite a bit, while Proposition 5.2 severely constrains possible torsion in the answer.

2. Lie algebra resolutions. If $a$ is a Lie algebra which is free over $\mathbf{Z}$, the standard free resolution of the trivial module $\mathbf{Z}$ over the enveloping algebra $\mathfrak{U}(\mathfrak{a})$ is the complex $R_{*}($ a $)$ with

$$
R_{k}(a)=\mathfrak{U}(a) \otimes_{\mathbf{z}} \Lambda^{k}(a)
$$


and differential $d: R_{k}(a) \rightarrow R_{k-1}($ a $)$ given by the formula

$$
\begin{aligned}
d\left(x \otimes x_{1} \wedge \cdots \wedge x_{k}\right)=\sum_{i=1}^{k}(-1)^{i+1}\left(x x_{i}\right) \otimes x_{1} \wedge \cdots \wedge \hat{x}_{i} \wedge \cdots \wedge x_{k} \\
\quad+\sum_{1 \leqslant i<j \leqslant k}(-1)^{i+j} x \otimes\left[x_{i}, x_{j}\right] \wedge x_{1} \wedge \ldots \wedge \hat{x}_{i} \wedge \ldots \wedge \hat{x}_{j} \wedge \ldots \wedge x_{k}
\end{aligned}
$$

(Here $\Lambda^{k}(\mathfrak{a})$ denotes the $k$ th exterior power of a over $\mathbf{Z}$.) We will let $C_{*}(a)$ denote $\mathbf{Z} \otimes_{\mathfrak{u}(a)} R_{*}(\mathfrak{a})$, so that the homology groups of $C_{*}(\mathfrak{a})$ are the Lie algebra homology groups $H_{*}(\mathfrak{a}, \mathbf{Z})$. The chain complex $C_{*}(a)$ is the standard Koszul complex [2, p. 290], with $C_{k}(a)=\Lambda^{k}(a)$ and $d: C_{k}(a) \rightarrow C_{k-1}(a)$ given by

$$
\begin{aligned}
d\left(x_{1}\right. & \left.\wedge \cdots \wedge x_{k}\right) \\
& =\sum_{1 \leqslant i<j \leqslant k}(-1)^{i+j}\left[x_{i}, x_{j}\right] \wedge x_{1} \wedge \cdots \wedge \hat{x}_{i} \wedge \cdots \wedge \hat{x}_{j} \wedge \cdots \wedge x_{k} .
\end{aligned}
$$

Now suppose that $g$ is a Lie algebra which is free over $\mathbf{Z}$, and that $a$ and $a^{\prime}$ are complementary subalgebras of $g$ in the sense that $a+a^{\prime}=g, a \cap a^{\prime}=\varnothing$. We will let $R_{*}\left(\mathfrak{g}, \mathfrak{a}^{\prime}\right)$ denote the $\mathfrak{U}(\mathfrak{g})$ chain complex given by

$$
R_{k}\left(g, a^{\prime}\right)=\mathfrak{u}(g) \otimes_{\mathfrak{u}\left(a^{\prime}\right)} \Lambda^{k}\left(g / a^{\prime}\right) .
$$

The differential $d: R_{k}\left(\mathrm{~g}, \mathrm{a}^{\prime}\right) \rightarrow R_{k-1}\left(\mathrm{~g}, \mathrm{a}^{\prime}\right)$ is determined by the formula

$$
\begin{aligned}
d(x & \left.\otimes x_{1} \wedge \cdots \wedge x_{k}\right)=\sum_{i=1}^{k}(-1)^{i+1}\left(x y_{i}\right) \otimes x_{1} \wedge \cdots \wedge \hat{x}_{i} \wedge \cdots \wedge x_{k} \\
& +\sum_{1 \leqslant i<j \leqslant k}(-1)^{i+j} x \otimes \pi\left[y_{i}, y_{j}\right] \wedge x_{1} \wedge \cdots \wedge \hat{x}_{i} \wedge \cdots \wedge \hat{x}_{j} \wedge \cdots \wedge x_{k}
\end{aligned}
$$

where $\pi: \mathfrak{g} \rightarrow \mathrm{g} / \mathrm{a}^{\prime}$ is the projection and $y_{i}$ in $\mathrm{g}$ stands for a representative of $x_{i}$ in $\mathrm{g} / \mathrm{a}^{\prime}[4, \mathrm{p} .40]$. The complex $R_{*}\left(\mathrm{~g}, \mathrm{a}^{\prime}\right)$ is a (generally nonfree) resolution of the trivial module $\mathbf{Z}$ over $\mathfrak{U}(g)$; in particular, $R_{*}\left(g, a^{\prime}\right)$ serves by restriction as a resolution of $\mathbf{Z}$ over $\mathfrak{U}(\mathfrak{a})$.

2.1 LEMMA. In the above situation, the natural map

$$
\mathfrak{U}(\mathfrak{a}) \otimes_{\mathbf{z}} \Lambda^{*}(\mathfrak{a}) \rightarrow \mathfrak{u}(g) \otimes_{\mathfrak{u}\left(a^{\prime}\right)} \Lambda^{*}\left(g / a^{\prime}\right)
$$

gives an isomorphism $R_{*}(\mathfrak{a}) \rightarrow R_{*}\left(\mathfrak{g}, \mathfrak{a}^{\prime}\right)$ of chain complexes over $\mathfrak{U}(\mathfrak{a})$.

Proof. See [4, Proposition 1.2].

2.2 Proposition. Suppose that $\mathrm{g}$ is a Lie algebra which is free over $\mathbf{Z}$ and that $\mathfrak{a}$ is a subalgebra of $\mathfrak{g}$ which possesses a complement. Then choice of a complement $\mathfrak{a}^{\prime}$ for $\mathfrak{a}$ determines an action of the center $\mathfrak{U}(\mathfrak{g})^{\mathfrak{g}}$ of $\mathfrak{U}(\mathfrak{g})$ on $R_{*}(\mathfrak{a})$. The induced action of $\mathfrak{U}(\mathfrak{g})^{\mathfrak{g}}$ on $H_{*}(\mathfrak{a}, \mathbf{Z})$ factors through the augmentation map $\varepsilon: \mathfrak{U}(\mathfrak{g})^{\mathfrak{g}} \rightarrow \mathbf{Z}$.

Proof. By 2.1 , choice of a complement $a^{\prime}$ determines a $\mathfrak{U}(\mathfrak{a})$-isomorphism $R_{*}(a) \cong R_{*}\left(g, a^{\prime}\right)$. Since $\mathfrak{u}(g)^{\mathfrak{g}}$ clearly acts on $R_{*}\left(g, a^{\prime}\right)$ in a way which commutes with the action of $\mathfrak{U}(\mathfrak{g})$ and thus of $\mathfrak{U}(\mathfrak{a})$, the first part of the proposition is immediate. Suppose that $\gamma \in \mathfrak{U}(\mathfrak{g})^{\mathfrak{g}}$. By elementary homological algebra, the $\mathfrak{U}(\mathfrak{a})$ 
chain homotopy class of the map $\gamma: R_{*}(\mathfrak{a}) \rightarrow R_{*}(\mathfrak{a})$ is determined by the induced map $H_{0}\left(R_{*}(\mathfrak{a})\right)=\mathbf{Z} \rightarrow H_{0}\left(R_{*}(\mathfrak{a})\right)=\mathbf{Z}$, which is multiplication by $\varepsilon(\gamma)$; this gives the second statement.

2.3 RemarK. We will apply 2.2 with $g=\mathrm{gl}_{n}(\mathbf{Z}), \mathfrak{a}=u_{n}$, and $\mathfrak{a}^{\prime}$ equal to the subalgebra of $g$ consisting of matrices which are zero in the strict upper triangle.

3. Gradings. Suppose that $W$ is an abelian group. An abelian group $G$ is graded by $W$ if for each $w \in W$ there is a subgroup $G_{w}$ of $G$ such that $G=\oplus_{w \in W} G_{w}$. The elements of $G_{w}$ are said to be homogeneous of degree $w$. A Lie algebra $a$ is graded by $W$ if $a$ is graded as an abelian group and if in addition, for each pair $u, v$ of elements of $W,\left[a_{u}, a_{v}\right] \subseteq a_{u+v}$.

If $a$ is a Lie algebra graded by $W$, then each abelian group $C_{k}(a)=\Lambda^{k}(a)$ in the chain complex $C_{*}(a)$ can also be graded by $W$ according to the usual convention that if $x_{i}$ is homogeneous of degree $w_{i}, 1 \leqslant i \leqslant k$, then $x_{1} \wedge \cdots \wedge x_{k}$ is homogeneous of degree $w_{1}+\cdots+w_{k}$. It is clear from the formula in $\$ 2$ that the differential $d$ of $C_{*}(a)$ respects these gradings, and therefore that the homology groups $H_{*}(\mathfrak{a}, \mathbf{Z})$ inherit a grading by $W$.

For each pair of integers $i, j$ with $1 \leqslant i<j \leqslant n$, let $e_{i, j} \in u_{n}$ be the matrix which is identically zero except for a one in row $i$, column $j$. Let $\phi_{i, j} \in \mathbf{Z}^{n}$ be the vector which is identically zero except for a one in coordinate $i$ and a -1 in cocrdinate $j$. We will use $\Phi$ to denote the set $\left\{\phi_{i, j}: 1 \leqslant i<j \leqslant n\right\}$.

3.1 Proposition. The Lie algebra $u_{n}$ is graded by $\mathbf{Z}^{n}$ in such a way that each matrix $e_{i, j}, 1 \leqslant i<j \leqslant n$, is homogeneous of degree $\phi_{i, j}$.

Proof. This follows immediately from a calculation with matrix commutators.

According to the remarks above, it is a consequence of 3.1 that $C_{*}\left(u_{n}\right)$ and $\left.H_{*}\left(u_{n}, \mathbf{Z}\right)\right)$ inherit a $\mathbf{Z}^{n}$-grading.

3.2 Proposition. If $\lambda=\left(\lambda_{1}, \ldots, \lambda_{n}\right) \in \mathbf{Z}^{n}$, then the homogeneous summands $C_{*}\left(u_{n}\right)_{\lambda}$ and $H_{*}\left(u_{n}, \mathbf{Z}\right)_{\lambda}$ vanish unless $1-i \leqslant \lambda_{i} \leqslant n-i$ and $\sum_{i=1}^{n} \lambda_{i}=0$.

Proof. Since $C_{*}\left(u_{n}\right)=\Lambda^{*}\left(u_{n}\right)$, it follows from the definition of exterior algebra that $C_{*}\left(u_{n}\right)_{\lambda}=0$ unless $\lambda=\sum_{\phi \in S} \phi$ for some subset $S$ of $\Phi$. This easily gives the desired condition.

\section{Two combinatorial lemmas.}

4.1 Lemma. Suppose that $p$ is a prime, $n \leqslant p+1$, and $a_{1}, \ldots, a_{n}$ are integers between -1 and $-n$ such that the sequences $\left(a_{1}, \ldots, a_{n}\right)$ and $(-1, \ldots,-n)$, when reduced $\bmod p$, differ by a permutation. Suppose also that $\sum_{i=1}^{n} a_{i}=\sum_{i=1}^{n}(-i)$. Then the sequences $\left(a_{1}, \ldots, a_{n}\right)$ and $(-1, \ldots,-n)$ differ by a permutation.

Proof. If $n \leqslant p$ the lemma is trivial; the summation condition is not necessary. If $n=p+1$, the apparent possibility that $-(p+1)$ appears twice in the list of $a_{i}$ 's is ruled out by the summation condition.

4.2 LemMA. For each permutation $\sigma$ in $S_{n}$, there is a unique subset $R_{\sigma}$ of $\Phi$ (see §3) such that $(\sigma(1), \ldots, \sigma(n))=(1, \ldots, n)+\sum_{\phi \in R_{o}} \phi$. 
Proof. Proceed by induction on $n$; the conclusion is obvious if $n=2$. In general, suppose that $\sigma(k)=1$, and note that in order for the desired equation to hold the set $R_{\sigma}$ must contain at least $k-1$ elements of $\Phi$ which have -1 in coordinate $k$. In fact, $\Phi$ contains exactly $k-1$ such elements; let $A$ be the collection of them. Inspection shows that

$$
(2, \ldots, k, 1, k+1, \ldots, n)=(1, \ldots, n)+\sum_{\phi \in A} \phi
$$

and induction shows that there is a unique subset $B$ of $\Phi$ containing vectors with zero in coordinate $k$ such that

$$
(\sigma(1), \ldots, \sigma(n))=(2, \ldots, k, 1, k+1, \ldots, n)+\sum_{\phi \in B} \phi .
$$

Set $R_{\sigma}=A \cup B$. This completes the proof.

5. Calculation of Lie algebra homology. In [3, p. 196] Carter and Lusztig exhibit $n$ elements $C_{1}, \ldots, C_{n}$ in the center of the enveloping algebra $\mathfrak{U}\left(\mathrm{gl}_{n}(\mathbf{Z})\right)$. Let $\gamma_{1}, \ldots, \gamma_{n}$ be the images of $C_{1}, \ldots, C_{n}$ under the automorphism of $\mathfrak{U}\left(\mathrm{gl}_{n}(\mathbf{Z})\right)$ induced by the automorphism of $\mathrm{gl}_{n}(\mathbf{Z})$ given by conjugation with the matrix of the permutation $\rho \in S_{n}$, where $\rho(i)=n+1-i$. Recall that $\varepsilon: \mathfrak{U}\left(\mathrm{gl}_{n}(\mathbf{Z})\right) \rightarrow \mathbf{Z}$ is the augmentation map.

5.1 Proposition. The elements $\gamma_{1}, \ldots, \gamma_{n}$ of the center of $\mathfrak{U}\left(\mathrm{gl}_{n}(\mathbf{Z})\right)$ have the following properties:

(1) $\varepsilon\left(\gamma_{k}\right)=s_{k}(-n, \ldots,-1)$, where $s_{k}$ is the kth elementary symmetric function in $n$ variables.

(2) The map $\gamma_{k}: C_{*}\left(u_{n}\right) \rightarrow C_{*}\left(u_{n}\right)$ provided by 2.3 preserves the grading of $C_{*}\left(u_{n}\right)$ by $\mathbf{Z}^{n}$ derived from 3.1 .

(3) If $v \in C_{*}\left(u_{n}\right)$ is homogeneous of degree $\lambda=\left(\lambda_{1}, \ldots, \lambda_{n}\right)$, then

$$
\gamma_{k}^{v}=s_{k}\left(\lambda_{1}-n, \ldots, \lambda_{n}-1\right) v \text {. }
$$

Proof. All three properties follow from an elementary calculation with the Carter-Lusztig formula.

5.2 Proposition. For each $\lambda=\left(\lambda_{1}, \ldots, \lambda_{n}\right) \in Z^{n}$, the homogeneous summand $H_{*}\left(u_{n}\right)_{\lambda}$ is annihilated by the greatest common divisor of the collection of numbers

$$
s_{k}\left(\lambda_{1}-n, \lambda_{2}-n+1, \ldots, \lambda_{n}-1\right)-s_{k}(-n,-n+1, \ldots,-1)
$$

as $k$ varies between 1 and $n$.

Proof. This is an immediate consequence of 2.2 and 5.1.

5.3 Proposition. The homogeneous summand $H_{*}\left(u_{n}, \mathbf{Z}_{(p)}\right)_{\lambda}$ is zero unless the polynomials $\Pi_{i=1}^{n}\left(x+\lambda_{i}-n+i+1\right)$ and $\Pi_{i=1}^{n}(x-n+i-1)$ are equal mod $p$, that is, unless the sequences $\left(\lambda_{1}-n, \ldots, \lambda_{n}-1\right)$ and $(-n, \ldots,-1)$, when reduced mod $p$, differ by a permutation.

Proof. This follows from the fact that the symmetric functions $s_{k}\left(a_{1}, \ldots, a_{n}\right)$ appear as coefficients of the polynomial $\left(x+a_{1}\right)\left(x+a_{2}\right) \cdots\left(x+a_{n}\right)$. 
5.4 CorollaRY. If $p \geqslant n-1$, then $H_{*}\left(u_{n}, \mathbf{Z}_{(p)}\right)_{\lambda}=0$ unless the sequences $\left(\lambda_{1}-1, \ldots, \lambda_{n}-1\right)$ and $(-n, \ldots,-1)$ differ by a permutation.

Proof. This is a combination of 3.2, 4.1, and 5.3.

PROOF of 1.3. By 5.4, if $H_{*}\left(u_{n}, \mathbf{Z}_{(p)}\right)_{\lambda} \neq 0$ then the sequences $\left(\lambda_{1}-n, \ldots, \lambda_{n}-1\right)$ and $(-n, \ldots,-1)$ differ by a permutation $\sigma \in S_{n}$. Adding $n+1$ to the elements involved shows that the sequences $\left(\lambda_{1}+1, \ldots, \lambda_{n}+n\right)$ and $(1, \ldots, n)$ differ by $\sigma$. By 4.2 , there is a unique subset $R_{\sigma}$ of $\Phi$ such that $\left(\lambda_{1}, \ldots, \lambda_{n}\right)=\sum_{\phi \in R_{o}} \phi$. This implies that $C_{*}\left(u_{n}\right)_{\lambda}=\Lambda^{*}\left(u_{n}\right)_{\lambda}$ is free of rank one (compare proof of 3.2) and therefore of necessity concentrated in a single dimension. It follows that the differential of $C_{*}\left(u_{n}\right)_{\lambda}$ is trivial, and that $H_{*}\left(u_{n}, \mathbf{Z}\right)_{\lambda}$ and $H_{*}\left(u_{n}, \mathbf{Z}_{(p)}\right)_{\lambda}$ are both free of rank one.

\section{REFERENCES}

1. F. Bachman and L. Grünenfelder, Über Lie-Ringe von Gruppen und ihre universellen Enveloppen, Comment. Math. Helv. 47 (1972), 332-340.

2. H. Cartan and S. Eilenberg, Homological algebra, Princeton Univ. Press, Princeton, N. J., 1956.

3. R. Carter and G. Lusztig, On the modular representations of the general linear and symmetric groups, Math. Z. 136 (1974), 193-242.

4. H. Garland and J. Lepowsky, Lie algebra homology and the MacDonald-Kac formula, Invent. Math. 34 (1976), 37-76.

5. L. Grünenfelder, On the homology of filtered and graded rings, J. Pure Appl. Algebra 14 (1979), 21-37.

6. B. Kostant, Lie algebra cohomology and the generalized Borel-Weil theorem, Ann. of Math. 74 (1961), 329-387.

7. L. Lambe and S. B. Priddy, Cohomology of nilmanifolds and torsion-free nilpotent groups, Northwestern University, preprint.

8. K. Nomizu, On the cohomology of compact homogeneous spaces of nilpotent Lie groups, Ann. of Math. 59 (1954), 531-538.

9. D. G. Quillen, Elementary proofs of some results of cobordism theory using Steenrod operations, Adv. in Math. 7 (1971), 29-56.

10. J. P. Serre, Algèbre locale multiplicités, Lecture Notes in Math., vol. 11, Springer, New York, 1975.

Department of Mathematics, University of Notre Dame, Notre Dame, Indiana 46556 